\title{
ON GROUP D.G. NEAR-RINGS
}

\author{
S. J. MAHMOOD AND J. D. P. MELDRUM
}

\begin{abstract}
Meldrum has generalized the idea of a group ring and has defined a group d.g. near-ring for a faithful d.g. near-ring $(R, S)$ on a multiplicative group $G$. In this paper we generalize this idea even further and define a group d.g. near-ring for an arbitrary d.g. near-ring. We also prove some results about the additive group of this d.g. near-ring similar to those proved by Meldrum for a group d.g. near-ring of a faithful d.g. near-ring.
\end{abstract}

1. Preliminaries. A set $R$, together with two binary operations + and $\cdot$, is called a (left) near-ring if:

(i) $(R,+)$ is a group (not necessarily abelian);

(ii) $(R, \cdot)$ is a semigroup;

(iii) $x(y+z)=x y+x z$ for all $x, y, z \in R$.

An element $d \in R$ is called distributive if $(x+y) d=x d+y d$ for all $x, y \in R$. The subset $D$ of distributive elements forms a subsemigroup of $(R, \cdot)$.

$R$ is called a distributively generated (d.g.) near-ring if $(R,+)$ is generated by a distributive semigroup $S$ which is not necessarily the whole set of distributive elements of $R$. A d.g. near-ring is denoted by $(R, S)$. We call $\theta:(R, S) \rightarrow(T, U)$ a d.g. near-ring homomorphism if $\theta:(R,+) \rightarrow(T,+)$ is a group homomorphism and $\theta:(R, \cdot) \rightarrow(T, \cdot)$ is a semigroup homomorphism such that $S \theta \subseteq U$. A semigroup homomorphism $\theta: S \rightarrow U$ is a d.g. near-ring homomorphism from $(R, S) \rightarrow(T, U)$ if and only if it is a group homomorphism from $(R,+)$ to $(T,+)$. From now on we will use the term homomorphism for a d.g. near-ring homomorphism unless otherwise stated.

If, for a group $G, \theta:(R, S) \rightarrow(E(G)$, End $G)$ is a homomorphism, then $\theta$ is called a d.g. representation of $(R, S)$ on $G$. Here $E(G)$ is the d.g. near-ring of mappings from $G$ to itself generated by End $G$, the set of all endomorphisms of $G$. A d.g. near-ring is called faithful if it has a faithful d.g. representation, i.e., $\operatorname{Ker} \theta=\{0\}$. Not all d.g. near-rings are faithful [3]. $(R, S)$ is faithful if and only if an identity 1 can be adjoined to $R$ such that the elements of $S$ remain distributive in the bigger d.g. near-ring [5]. However, with every d.g. near-ring we can associate two faithful d.g. near-rings (Meldrum [3], Mahmood [2]). The upper faithful d.g. near-ring for $(R, S)$ is a faithful d.g. near-ring $(\bar{R}, S)$ together with an epimorphism $\theta$ : $(\bar{R}, S) \rightarrow(R, S)$ such that (i) $\left.\theta\right|_{S}=1_{S}$, (ii) if $\phi:(T, U) \rightarrow(R, S)$ is a homomorphism, where $(T, U)$ is faithful, then there exists a unique homomorphism $\psi$ : $(T, U) \rightarrow(\bar{R}, S)$ such that $\psi \theta=\phi$. The lower faithful d.g. near-ring for $(R, S)$ is a 
faithful d.g. near-ring $(\underline{R}, S)$ together with an epimorphism $\underline{\theta}:(R, S) \rightarrow(\underline{R}, \underline{S})$ such that (i) $S \underline{\theta}=\underline{S}$, (ii) if $\phi:(R, S) \rightarrow(T, U)$ is a homomorphism, where $(T, U)$ is faithful, then there exists a unique homomorphism $\psi:(\underline{R}, \underline{S}) \rightarrow(T, U)$ such that $\underline{\theta} \psi=\phi$.

We will be using the following two results from [3]. For each $(R, S)$ group $H$, $\operatorname{Ker} \underline{\theta} \subseteq \operatorname{Ann}_{R} H$. Let $X \subseteq R$, where $(R, S)$ is a d.g. near-ring. Then $\operatorname{Id}\langle X\rangle$, the ideal generated by $X$, is the normal subgroup of $(R,+)$ generated by $R X S=$ $\{r x s, r x, x s, x ; r \in R, x \in X, s \in S\}$. We now present basic facts about d.g. nearrings taken from [4]. Let $(R, S)$ be a faithful d.g. near-ring and let $G$ be a multiplicative group. Let $X$ be any set, $Y=X \times G=\{(x, g) ; x \in X, g \in G\}$, and $F=\operatorname{Fr}(Y, R, S)$ be the free $(R, S)$ group on the set $Y$ [3]. Then by means of right regular representations, $G$ can be defined as a group of $(R, S)$ automorphisms of $F$. So the semigroup $S G=\{s g ; s \in S, g \in G\}$ of endomorphisms of $F$ generates a d.g. near-ring $(R(G), S G)$ in $E(F)$. This d.g. near-ring is defined to be the group d.g. near-ring of $(R, S)$ on $G$. Also $r g=g r$ in $R(G)$ for all $r \in R, g \in G . F$ is the free $(R(G), S G)$ group on the set $X$ and $(R(G),+)$ considered as an $(R, S)$ group is an orthogonal sum of its $(R, S)$ subgroups $\{R g ; g \in G\}$.

The idea of an orthogonal sum comes from Fröhlich [1]. If $\left\{H_{\lambda} ; \lambda \in \Lambda\right\}$ is a family of $(R, S)$ groups, then $H$ is an orthogonal sum of $\left\{H_{\lambda} ; \lambda \in \Lambda\right\}$ if it is an $(R, S)$ group, and $(R, S)$ homomorphisms $\alpha_{\lambda}, \beta_{\lambda}$ exist for all $\lambda \in \Lambda$ such that $\alpha_{\lambda}$ : $H_{\lambda} \rightarrow H, \beta_{\lambda}: H \rightarrow H_{\lambda}$ and $\alpha_{\lambda} \beta_{\mu}$ is the identity map on $H_{\lambda}$ if $\lambda=\mu$, and is the zero map otherwise. Note that this forces $\alpha_{\lambda}$ to be a monomorphism and $\beta_{\lambda}$ an epimorphism for all $\lambda \in \Lambda$. We add the condition that $H=G p\left\langle H_{\lambda} \alpha_{\lambda} ; \lambda \in \Lambda\right\rangle$. Fröhlich calls this a covered orthogonal sum. This is equivalent to saying that there exist homomorphisms $\theta, \phi *{ }_{\lambda \in \Lambda} H_{\lambda} \stackrel{\theta}{\rightarrow} H \stackrel{\phi}{\rightarrow} \bigoplus_{\lambda \in \Lambda} H_{\lambda}$, where $*$ indicates the free $(R, S)$ product, $\oplus$ indicates the direct sum, and $\theta, \phi$ are epimorphisms which respect the injection of $H_{\lambda} \rightarrow{ }_{\lambda \in \Lambda} H_{\lambda}$ and the projection $\bigoplus_{\lambda \in \Lambda} H_{\lambda} \rightarrow H_{\lambda}$.

2. The group d.g. near-ring. Let $(R, S)$ be an arbitrary d.g. near-ring and $G$ a multiplicative group. Let $(\bar{R}, S)$ be the upper faithful d.g. near-ring for $(R, S)$ together with the natural homomorphism $\theta:(\bar{R}, S) \rightarrow(R, S)$. Since $(\bar{R}, S)$ is faithful we can construct $(\bar{R}(G), S G)$. Let $I=\operatorname{Ker} \theta$ and $I G=\{a g ; a \in I, g \in G\}$. Denote by $J$ the ideal $\operatorname{Id}\langle I G\rangle$. By the remark above, $J$ is the normal subgroup of $(\bar{R}(G),+)$ generated by

$$
\begin{aligned}
\bar{R}(G) I G S G=\left\{\left(\sum r_{i} g_{i}\right)(a g)(s h),\left(\sum r_{i} g_{i}\right)(a g),(a g)(s h), a g ;\right. & \\
& \left.\sum r_{i} g_{i} \in \bar{R}(G), a \in I, s \in S, g, h \in G\right\} .
\end{aligned}
$$

Using results about $\bar{R}(G)$ from [4], we have

$$
\bar{R}(G) I G S G=\left\{\left(\sum r_{i} g_{i}(a g), a g ; \sum r_{i} g_{i} \in \bar{R}(G), a \in I, g \in G\right\}\right.
$$

since $I$ is an ideal of $\bar{R}$.

Definition 1. ( $\bar{R}(G), S G) / J$ is called the group d.g. near-ring of $(R, S)$ on $G$.

Without danger of confusion we may denote it $(R(G), S G)$, as we will see later that $S G+J / J$ is naturally isomorphic to $S G$. The following generalization of 
Fröhlich's result-if $(R, S)$ is a d.g. near-ring and $H$ is an $S$ group, then $H$ is an $(R, S)$ group provided $\operatorname{Ker} \pi \subseteq \operatorname{Ann}_{\mathrm{Fr}(S)} H$, where $\pi$ is the natural homomorphism from the free d.g. near-ring $(\operatorname{Fr}(S), S)$ on $S$ to $(R, S)$-is needed for our first result.

Lemma 2. Let $\phi:(R, S) \rightarrow(T, U)$ be an epimorphism, and let $H$ be an $(R, S)$ group which is also a $U$ group. Then $H$ is a $(T, U)$ group if $\operatorname{Ker} \phi \subseteq \operatorname{Ann}_{R} H$.

Proof. Let $\psi$ be the representation of $(R, S)$ on $H$. By the hypothesis, $\operatorname{Ker} \phi \subseteq$ Ker $\psi$. Hence $\psi$ factors through $\phi$ giving a representation of $(T, U)$ on $H$.

TheOREM 3. $(\bar{R}(G) / J,+)$ is an $(R, S)$ group.

Proof. Clearly $(\bar{R}(G) / J,+)$ is an $(\bar{R}, S)$ group. Let $\sum r_{i} g_{i}+J \in \bar{R}(G) / J, a \in I$. Then

$$
\left(\sum r_{i} g_{i}+J\right) a=\left(\sum r_{i} g_{i}\right) a+J=J
$$

since $a \in I$ and $J \supseteq I G$. Therefore $I \subseteq \operatorname{Ann}_{R}^{-}(\bar{R}(G) / J)$. Hence $(\bar{R}(G) / J,+)$ is an $(R, S)$ group by Lemma 2 .

We note that

$$
(\bar{R}(G) / J,+)=G p\langle(\bar{R} g+J) / J ; g \in G\rangle,
$$

where each $(\bar{R} g+J) / J$ is an additive subgroup of $\bar{R}(G) / J$. Moreover, the groups $\{(\bar{R} g+J) / J ; g \in G\}$ can be considered as $(R, S)$ groups in a natural way, as in Theorem 3.

We now look at the relationship of $(R(G), S G)$ to $(\underline{R}(G), \underline{S} G)$. Let $(\underline{R}, \underline{S})$ be the lower faithful d.g. near-ring for $(R, S)$ together with the natural homomorphism $\underline{\theta}$ : $(R, S) \rightarrow(\underline{R}, \underline{S})$. Then as before we can construct the group d.g. near-ring ( $\underline{R}(G), \underline{S} G)$ which is a sub-d.g. near-ring of $(E(\underline{F})$, End $\underline{F})$, where $\underline{F}=\operatorname{Fr}(Y, \underline{R}, \underline{S})$, the free $(\underline{R}, \underline{S})$ group on $Y$. Let $\bar{F}=\operatorname{Fr}(Y, \bar{R}, S)$ be the free $(\bar{R}, S)$ group on $Y$. Clearly, $(\bar{R}(G), S G)$ is a sub-d.g. near-ring of $(E(\bar{F})$, End $\bar{F})$. By the freeness of $\bar{F}$ there exists a unique $(\bar{R}, S)$ homomorphism $\mu: \bar{F} \rightarrow \underline{F}$ which extends the identity map on $Y$. Note that since $(\underline{R}, \underline{S})$ is a homomorphic image of $(\bar{R}, S)$ under $\theta \underline{\theta}, \underline{F}$ is an $(\bar{R}, S)$ group in a natural way. We have

$$
((x, g) r) \mu=(x, g)(r \theta \underline{\theta}) \quad \text { for all }(x, g) \in Y, r \in \bar{R} \text {. }
$$

LEMMA 4. $\alpha: S G \rightarrow \underline{S} G$ defined by $s g \rightarrow(s \theta \underline{\theta}) g$ extends to a homomorphism from $(\bar{R}(G), S G)$ to $(\underline{R}(G), \underline{S} G)$.

Proof. $\alpha$ is certainly a semigroup homomorphism $S G \rightarrow \underline{S} G$, so we need only check that it extends to a group homomorphism $(\bar{R}(G),+) \rightarrow(\underline{R}(G),+)$, which we will also denote by $\alpha$.

Let $r=\varepsilon_{1} s_{1} g_{1}+\cdots+\varepsilon_{n} s_{n} g_{n}=0$ in $\bar{R}(G)$, where $\varepsilon_{i}= \pm 1, s_{i} \in S, g_{i} \in G$. Then

$$
r \alpha=\varepsilon_{1}\left(s_{1} \theta \underline{\theta}\right) g_{1}+\cdots+\varepsilon_{n}\left(s_{n} \theta \underline{\theta}\right) g_{n}
$$

has to be shown to be 0 in $\underline{R}(G)$. Since $\underline{F}$ is the free $(\underline{R}(G), \underline{S} G)$ group on $\{(x, 1) ; x \in X\}$, we need only show that $(x, 1) r=0$ for all $x \in X$. But $(x, 1) r=0$ 
for all $x \in X$ in $\bar{F}$. Hence

$$
\begin{aligned}
0 & =((x, 1) r) \mu=\left((x, 1)\left(\varepsilon_{1} s_{1} g_{1}+\cdots+\varepsilon_{n} s_{n} g_{n}\right)\right) \mu \\
& =\left(\varepsilon_{1}\left(x, g_{1}\right) s_{1}+\cdots+\varepsilon_{n}\left(x, g_{n}\right) s_{n}\right) \mu \\
& =\left(\varepsilon_{1}\left(x, g_{1}\right) s_{1}\right) \mu+\cdots+\left(\varepsilon_{n}\left(x, g_{n}\right) s_{n}\right) \mu \quad \text { since } \mu \text { is a homomorphism } \\
& =\varepsilon_{1}\left(x, g_{1}\right)\left(s_{1} \theta \underline{\theta}\right)+\cdots+\varepsilon_{n}\left(x, g_{n}\right)\left(s_{n} \theta \underline{\theta}\right) \\
& =\varepsilon_{1}(x, 1)\left(s_{1} \theta \underline{\theta}\right) g_{1}+\cdots+\varepsilon_{n}(x, 1)\left(s_{n} \theta \underline{\theta}\right) g_{n} \\
& =(x, 1)\left(\varepsilon_{1}\left(s_{1} \theta \underline{\theta}\right) g_{1}+\cdots+\varepsilon_{n}\left(s_{n} \theta \underline{\theta}\right) g_{n}\right)=(x, 1)(r \alpha) .
\end{aligned}
$$

This suffices to prove the result.

THEOREM 5. ( $\underline{R}(G), \underline{S} G)$ is a homomorphic image of $(\bar{R}(G), S G) / J$.

Proof. It suffices to show that $J \subseteq \operatorname{Ker} \alpha$. Let $a g \in I G$. Then $(a g) \alpha=(a \theta \underline{\theta}) g=$ $(0 \underline{\theta}) g=0$ in $\underline{R}(G)$. Hence $I G \subseteq \operatorname{Ker} \alpha$, and so $J \subseteq \operatorname{Ker} \alpha$, since $J=\operatorname{Id}\langle I G\rangle$ and $\operatorname{Ker} \alpha$ is an ideal.

We thus have the following commutative diagram:

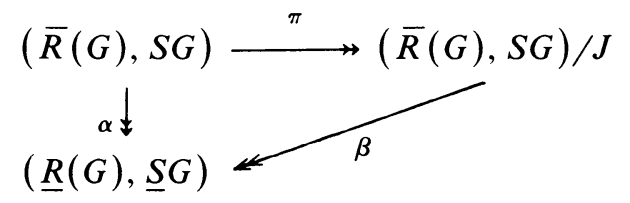

Here $\pi$ is the natural homomorphism. Note that $\beta$ is uniquely defined.

We now wish to show that $(R(G), S G)$ is an orthogonal sum of $(R, S)$ groups $(R g,+)$ each isomorphic to $(R,+)$.

THEOREM 6. $(\bar{R}(G), S G) / J$ is an orthogonal sum of $(R, S)$ groups $(R g,+)$ each isomorphic to $(R,+)$.

Proof. Consider the following diagram:

$$
\begin{array}{ccccccc}
\bar{R} g & \stackrel{\alpha_{g}}{\rightarrow} & (\bar{R}(G), S G) & \stackrel{\psi}{\rightarrow} & \bigoplus_{g \in G} \bar{R} g & \stackrel{\beta_{g}}{\rightarrow} & \bar{R} g \\
\downarrow \theta_{g} & & \downarrow \pi & & \downarrow \bar{\theta} & & \downarrow \theta_{g} \\
R g & \overrightarrow{\delta_{g}} & (\bar{R}(G), S G) / J & \vec{\phi} & \bigoplus_{g \in G} R g & \overrightarrow{\gamma_{g}} & R g
\end{array}
$$

$R g$ is an $(R, S)$ group, hence an $(\bar{R}, S)$ group, which is isomorphic to $(R,+)$ and whose elements are $\{r g: r \in R\}$. The maps $\theta_{g}, \bar{\theta}, \theta_{g_{-}}$are the obvious $(\bar{R}, S)$ homomorphisms induced by $\theta$. Note $\operatorname{Ker} \theta_{g}=I g, \operatorname{Ker} \bar{\theta}=\bigoplus_{g \in G} I g$. Finally $\pi$ is the canonical homomorphism, $\alpha_{g}, \beta_{g}$ and $\psi$ are the maps arising from the orthogonal sum properties of $\bar{R}(G)$, and $\gamma_{g}$ are the usual projections. We wish to show the existence of homomorphisms $\delta_{g}, \phi$ making the diagram commutative. Note that the right-hand square is commutative, as can be seen from the definitions of the maps. So $\beta_{g} \theta_{g}=\bar{\theta} \gamma_{g}$. 
Consider $I g \subseteq \bar{R}(G)$ for some $g \in G$. Then $(I g) \psi \subseteq I g \subseteq \bar{R} G \subseteq \bigoplus_{g \in G} \bar{R} g$, from the definition of $\psi$. Hence $(I g) \psi \subseteq \operatorname{Ker} \bar{\theta}$. This holds for all $g \in G$. Thus $I G \subseteq$ $\operatorname{Ker} \psi \overline{\boldsymbol{\theta}}$. Since $\operatorname{Ker} \psi \overline{\boldsymbol{\theta}}$ is an ideal, it follows that $J=\operatorname{Id}\langle I G\rangle \subseteq \operatorname{Ker} \psi \overline{\boldsymbol{\theta}}$. So $\psi \overline{\boldsymbol{\theta}}$ factors uniquely through $\pi$, i.e., there exists $\phi:(\bar{R}(G), S G) / J \rightarrow \oplus_{g \in G} R g$ such that $\pi \phi=\psi \bar{\theta}$ and $\phi$ is unique. So $\phi$ exists and the middle square is commutative. It follows that $\operatorname{Ker} \pi \phi \cap \bar{R} g=I g$. This leads to the following result, which we state separately.

LEMMA 7. In $\bar{R}(G), \bar{R} g \cap J=$ Ig for all $g \in G$.

We return to the proof of Theorem 6. Consider $\alpha_{g} \pi$. By the definition of orthogonal sum, $\alpha_{g}$ is a monomorphism. So

$$
\operatorname{Ker} \alpha_{g} \pi=\alpha_{g}^{-1}\left(\operatorname{Ker} \pi \cap \operatorname{Im} \alpha_{g}\right)=\alpha_{g}^{-1}(J \cap \bar{R} g)^{*}=I g \subseteq \bar{R} g
$$

by Lemma 7. But $\operatorname{Ker} \theta_{g}=I g$. So there exists a unique monomorphism $\delta_{g}: R g \rightarrow$ $(\bar{R}(G), S G) / J$ such that $\theta_{g} \delta_{g}=\alpha_{g} \pi$. In particular, $\delta_{g}: R G \rightarrow \bar{R} g+J / J$. Also, $\phi:$ $\bar{R} g+J / J \rightarrow R g \subseteq \oplus_{g \in G} R g$. The complete diagram is commutative and $(\bar{R}(G), S G) / J$ is an orthogonal sum of the groups $\bar{R} g+J / J$, each of which is isomorphic to $(R,+)$.

COROllaRY 8. $(\bar{R} g+J) / J \cong(R g,+) \cong(R,+)$ as $(R, S)$ group for each $g \in G$.

Note that Lemma 7 implies that $J \cap S G$ is trivial and, hence, that $S G+J / J \cong S G$ as a semigroup. So we can write $(R(G), S G)$ for $(\bar{R}(G), S G) / J$, and we can identify $S G+J / J$ with $S G, \bar{R} g+J / J$ with $R g$ for each $g \in G$.

We note that for any group d.g. near-ring $(R(G), S G)$, the subnear-ring $\left(R 1_{G}, S 1_{G}\right)$ is naturally isomorphic to $(R, S)$. Since a sub-d.g. near-ring of a faithful d.g. near-ring is faithful, it follows that if $(R, S)$ is not faithful, then neither is $(R(G), S G)$ for any group $G$. We do have a faithful d.g. near-ring with a projection on to $(R(G), S G)$, namely $\pi:(\bar{R}(G), S G) \rightarrow(R(G), S G)$. We also have a faithful d.g. near-ring which is a homomorphic image of $(R(G), S G)$, namely $\beta$ : $(R(G), S G)$ $\rightarrow(\underline{R}(G), \underline{S} G)$. We now relate these to the upper and lower faithful d.g. near-rings for $(R, S)$.

THEOREM 9. Let $\phi:(U, S G) \rightarrow(R(G), S G)$ be the upper faithful d.g. near-ring for $(R(G), S G)$. Then $(U, S G)$ is an orthogonal sum of the $(\bar{R}, S)$ groups $\{(\bar{R} g,+) ; g \in$ $G\}$ and the canonical homomorphism $\psi:(\bar{R}(G), S G) \rightarrow(U, S G)$ such that $\psi \phi=\pi$ respects the orthogonal sum structure.

In Fröhlich's notation, $\psi$ is an orthogonal homomorphism.

Proof. Consider the following commutative diagram:

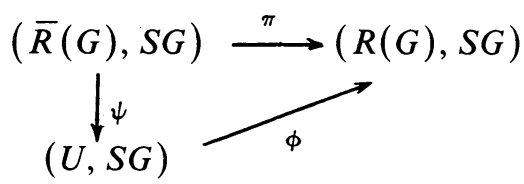

where all the maps are epimorphisms and restrict to the identity on $S G$. Denote by $(T g, S g)$ the sub- $(\bar{R}, S)$ group of $(U,+)$ generated by $(S g) \psi$, which we identify with $S g$. Then $\left(T 1_{G}, S 1_{G}\right)$ is a d.g. near-ring. It is faithful, as it is a sub-d.g. near-ring of a 
faithful d.g. near-ring. From the properties of upper faithful d.g. near-rings, it follows that $\left(T 1_{G}, S 1_{G}\right) \cong\left(\bar{R} 1_{G}, S 1_{G}\right)$. Hence, $(T g, S g)=\left(T 1_{G}, S 1_{G}\right) g \cong(\bar{R} g, S g)$. Further, $\psi: \bar{R} g \rightarrow T g$ and is the identity on $S g \rightarrow S g$. Thus Ker $\psi \cap \overline{R g}=\{0\}$. This leads to an embedding of $(\bar{R} g, S g)$ in $(U, S G)$ for each $g \in G$.

We now need projections of $(U, S G)$ onto $(\bar{R} g, S g)$ for each $g \in G$. With a change in notation, we use the proof of Theorem 6 to obtain the following commutative diagram:

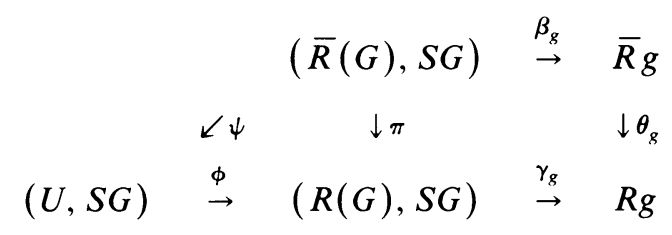

For $g=1_{G}$ we have a map $\phi \gamma_{1}:(U, S G) \rightarrow R 1_{G}$, and $\phi \gamma_{1}$ maps $\left(T 1_{G}, S 1_{G}\right) \rightarrow$ $\left(R 1_{G}, S 1_{G}\right)$. As before, it follows that $\phi \gamma_{1}$ restricted to $T 1_{G}$ factors through $\theta_{1}$ : $\bar{R} 1_{G} \rightarrow R 1_{G}$, using the properties of upper faithful d.g. near-rings. Now right multiplication by $g$ maps $T 1_{G}$ to $T g$ and $R 1_{G}$ to $R g$. Hence, $\phi \gamma_{g}$ always factors through $\theta_{g}$, when restricted to $T g$. This finishes the proof of the result.

THEOREM 10. Let $\phi:(R(G), S G) \rightarrow(\underline{U}, \underline{S} \underline{G})$ be the lower faithful d.g. near-ring for $(R(G), S G)$. Then $(\underline{\bar{U}}, \underline{S} \underline{G})$ is an orthogonal sum of the $(\underline{R}, \underline{S})$ groups $\{(\underline{R} g,+) ; g \in$ $G\}$ and the canonical homomorphism $\psi:(\underline{U}, \underline{S} \underline{G}) \rightarrow(\underline{R}(G), \underline{S} G)$ such that $\beta=\underline{\phi} \psi$ respects the orthogonal sum structure.

Proof. The proof parallels that of Theorem 9 fairly closely. So we will only give an outline. Consider the following commutative diagram:

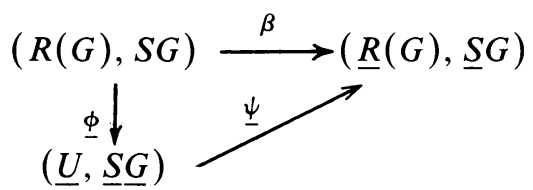

Denote by $(\underline{T g}, \underline{S g})$ the sub- $(\underline{R}, \underline{S})$ group of $(\underline{U},+)$ generated by $(S g) \phi$. Considering first $\underline{T} 1_{G}$, we see as before that $\left(\underline{T} 1_{G}, \underline{S} 1_{G}\right) \cong\left(\underline{R} 1_{G}, \underline{S} 1_{G}\right)$. This justifies the assumption made in the statement of the theorem that $(S G) \phi=\underline{S} G$. Using right multiplication by $g$ gives us $(\underline{T} g, \underline{S g}) \cong(\underline{R g}, \underline{S g})$, and we have the embedding of $(\underline{R g}, S g)$ in $(\underline{U}, \underline{S} \underline{G})$ for each $g \in G$.

For the second part, we have an easier situation. From above, we know that $\psi$ respects the embeddings. Since $(\underline{R}(G), \underline{S} G)$ is an orthogonal sum of $\{(\underline{R g}, \underline{S} g) ; g \bar{\epsilon}$ $G\}$, and $(\underline{U}, \underline{S} \underline{G})$ is mapped onto it by a homomorphism $\psi$ respecting the embeddings, it follows that $(\underline{U}, \underline{S} \underline{G})$ is an orthogonal sum of $\{(\underline{R} g, \underline{S g}) ; g \in G\}$, and $\underline{\psi}$ respects the orthogonal sum structure.

The next theorem follows immediately.

THEOREM 11. If $(\underline{R}(G), \underline{S} G)$ is the free $(\underline{R}, \underline{S})$ sum of $\{(\underline{R} g, \underline{S} g) ; g \in G\}$, then $\beta$ : $(R(G), S G) \rightarrow(\underline{R}(G), \underline{S} G)$ is the lower faithful d.g. near-ring for $(R(G), S G)$. 
If $(U, S G)$ is the free $(\bar{R}, S)$ sum of $\{(\bar{R} g, S g) ; g \in G\}$, then $\pi:(\bar{R}(G), S G) \rightarrow$ $(\bar{R}(G), S G)$ is the upper faithful d.g. near-ring for $(R(G), S G)$, and $(\bar{R}(G), S G)$ is the free $(\bar{R}, S)$ sum of $\{(\bar{R} g, S g) ; g \in G\}$.

The concrete determination of the upper and lower faithful d.g. near-rings for a given d.g. near-ring is difficult and involves a good deal of group theory in the form of group presentations in all cases covered so far. The case of lower faithful d.g. near-rings has been treated in [5] and that of upper faithful d.g. near-rings in [6]. In [6] the near-rings considered in detail are the zero near-rings on the finite dihedral groups. The smallest example of a group d.g. near-ring, namely $(R(G), S G)$ for $(R,+)$ the dihedral group of order 6 and $G$ the cyclic group of 2 , needs a very sophisticated group theoretic treatment, as anyone who consults [5 or 6] can see. So we are not in a position to give details here. But we hope to examine this situation in some detail in a later paper.

There are some interesting questions which arise from the last two theorems. When is $(\bar{R}(G), S G)$ the upper faithful d.g. near-ring for $(R(G), S G)$ ? And when is $(\underline{R}(G), \underline{S} G)$ the lower faithful d.g. near-ring for $(R(G), S G)$ ? These seem to be hard questions whose answer will depend on a detailed knowledge of the structure of the corresponding groups. This is also true of the problem of giving an "interval" characterization of $(R(G), S G)$, that is, one that does not involve going through $(\bar{R}, S)$.

Finally, a comment about group near-rings for arbitrary near-rings: The structure of a group near-ring is closely related to the free near-ring-module product. In the case of a zero-symmetric near-ring, this product exists, as general theorems about free products in varieties assure us. But detailed structural results are only emerging now, and they lead to a very complicated structure. Again it is hoped that these results will be followed up at a later stage.

\section{REFERENCES}

1. A. Fröhlich, On groups over a d.g. near-ring. I: Sum constructions and free R-groups, Quart. J. Math. Oxford (2) 11 (1960), 193-210.

2. S. J. Mahmood, Limits and colimits in categories of d.g. near-rings, Proc. Edinburgh Math. Soc. 23 (1980), 1-7.

3. J. D. P. Meldrum, The representation of d.g. near-rings, J. Austral. Math. Soc. 16 (1973), 467-480.

4. __. The group distributively generated near-ring, Proc. London Math. Soc. (3) 32 (1976), $323-346$.

5. __ Presentations of faithful d.g. near-rings, Proc. Edinburgh Math. Soc. 23 (1980), 49-56.

6. Upper faithful d.g. near-rings (submitted).

DePartment of Mathematics, Quaid-I-Azam University, Islamabad, Pakistan

Department of Mathematics, University of Edinburgh, Edinburgh EH9 3JZ, Scotland 